\title{
NOTES
}

\section{UN CONTREPOIDS DE PRESSOIR À HUILE D'ENTREMONT (Bouches-du-Rhône)}

\author{
par Christian GOUDINEAU
}

En 1968, dans le tome XXVI de Gallia, Fernand Benoît rendait comple de vingt années de fouilles à Entremont ${ }^{1}$. Dans les pages qu'il consacrait à l'artisanat agricole (p. 18-19), il signalait la trouvaille de "dalles d'areae de pressoirs" à huile et de "contrepoids à rainure, tous incomplets ». Il ajoutait ceci : " la mauvaise qualité de la roche locale (oligocène) avait rendu nécessaire la recherche de pierres de grande taille des carrières aixoises de Bibemus et de poudingue de Beauregard pour la confection de plusieurs areae et de presque tous les contrepoids $\|^{2}$. Dans cette rédaction, la phrase semblait suggérer que quelques areae et au moins un contrepoids avaient été taillés dans la pierre locale, en dépit de sa mauvaise qualité. C'est bien le cas, en effet, pour des areae. En revanche, si un contrepoids fait exception. c'est pour la raison que le matériau dont il est fait ne provient ni du plateau d'Entremont ni même de la région aixoise.

Cette pièce a élé trouvée dans la salle 8 de l'îlot XI (cf. le plan publiẻ en 1968). On l'y voit toujours bien qu'elle ait été déplacée vers le mur Nord de la case (fig. 1). C'est manifeste-

1 F. Benoit, "Les résultats historiques des fouilles d'Entremont, 1946-1967", Gallia, 26, 1968, p. 1-31.

2 Dans son guide, Entremont, Gap, 1969, qui s'inspire largement de l'article de Gallia, la phrase se termine ainsi : "... pour la confection des areae et des contrepoids *. ment un remploi, et en cela elle a subi le sort des autres contrepoids connus à Entremont : alors que certaines dalles d'areae sont demeurées en place, ils ont été endommagés et dispersés. Cinq d'entre eux, après avoir été extirpés des salles qui les abritaient, furent jetés à la rue où on les laissa; un sixième fut réutilisé pour obturer l'entrée d'une case (îlot XI, pièce 9) ; trois autres, enfin, dont celui qui nous intéresse, servirent (selon F. Benoît) d'assise à un poteau destiné à soutenir la toiture d'une pièce.

Il ne s'agit pas ici d'étudier ce bloc en tant qu'élément d'une huilerie protohistorique ${ }^{3}$, mais d'attirer l'attention sur la technique particulière qui a présidé à sa taille et qui reste bien visible sur les deux faces qui n'ont pas été affectées par la rainure et les mortaises destinées à la fixation de la machinerie (fig. 2). C'est la taille layée en chevrons opérée au marteau taillant (fig. 3), bien décrite par J.-Cl. Bessac ${ }^{4}$ et considérée à juste titre comme caractéristique des ateliers massaliotes (fig. 4) : on en voit les traces sur les carreaux des remparts hellénis-

3 On se reportera à la thèse de $3^{\text {e }}$ cycle récemment soutenue d̀ \ix par J.-P. Brun, Recherches sur les huileries antiques dans le Var, qui déborde largement Ir cadre géographique énoncé par le titre. I paraître ('n supplément de la R.A.X.

4 J.-Cl. Bessac, "Le rempart hellénistique de Saint-Blaise, technique de construction ", Documents d'Archéologie . Iéridionale, 3, 1980, p. 145. 


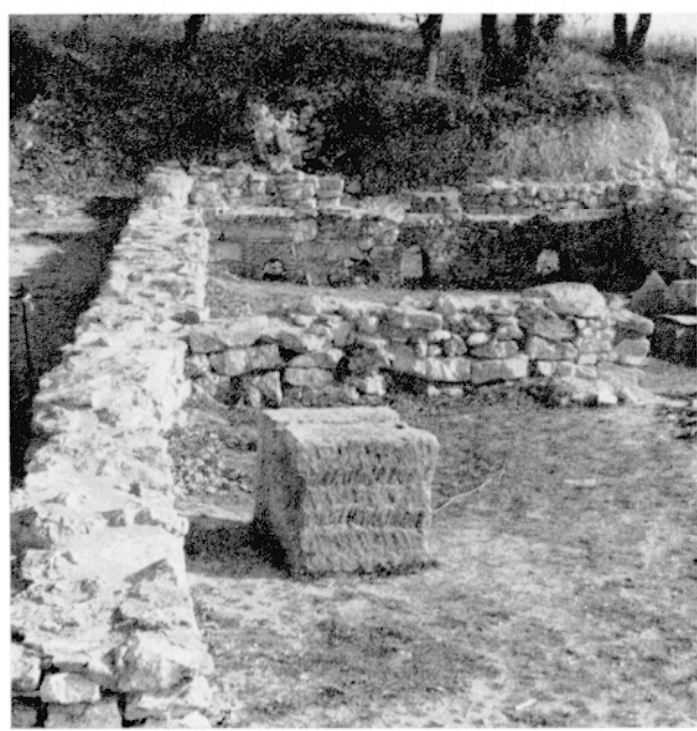

1 Situation du contrepoids. Au fond, la salle à trois fours 'clicho de l'auleur.

tiques de Marseille et de Saint-Blaise ${ }^{5}$. Cette observation et ce rapprochement suggéraient une analyse du matériau. Celle-ci, exécutée par le Laboratoire de Géologie historique et de Paléontologie de Marseille ${ }^{6}$, conclut sans ambiguïté : la pierre provient des carrières du Cap Couronne, celles-là même qui ont fourni les blocs du rempart de Marseille.

Ces données appellent plusieurs réflexions. La premiere purement technique : J.-Cl. Bessac: se demandait, ¿ propos du rempart de SaintBlaise, si la préparation des blocs, layage compris, s'étail effectuée à la carrière ou «au

5 Inutile de préciser que $F$. Benoil etait asse\% averti des réalités régionales pour avoir fait cotle observation. Son silence surprend: peut-être se reservait-il de signaler celte diconverle avec un certain éclat, ce dont sa mort, survenue au début de 1969 , l'aurait empêché ?

6 Rapport de IIme Fr. Catzigras (que nous remercions ici) : "calcaire à accumulation de débris organiques dont des mélobésiées, des bryozoaires el des lamellibranches. Microfacies : calcaire bioclastique à ciment sparitique, grains de quartz, à nombreux débris organiques représentant, par ordre d'abondance, des bryozoaires, des crustacés, des échinides, des lamellibranches, des mélobésiées, quelques foraminiferes et spicules d'éponge. Cel echantillon appartient à un bloc de "pierre de la Couronne" d'âge miocène (burdigalien supérieur;" ".

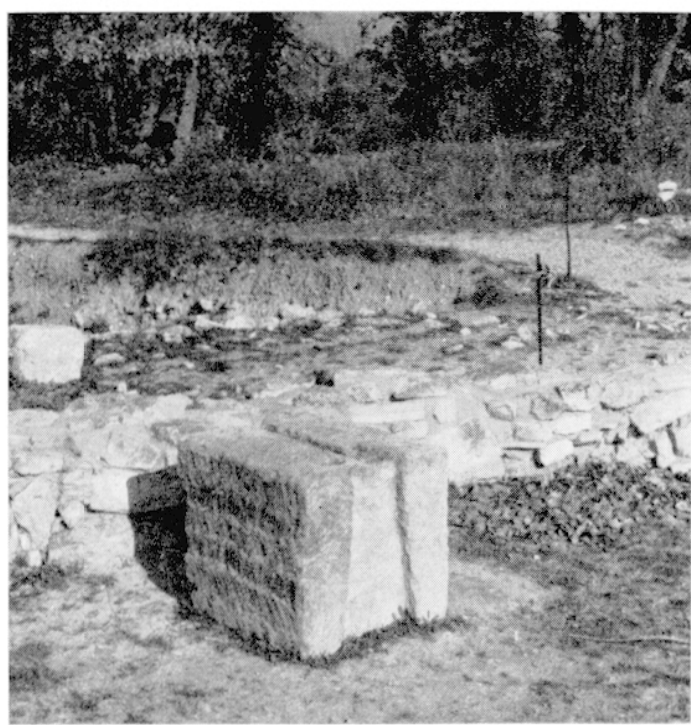

2 Le contrepoids avec les rainures caractéristiques pour la fixation du trenil cliche de l'auteur.

pied du mur" et il penchait pour la seconde solution?. Jans le cas d'lintremont, la probabilité est faible que le bloc ait été préparé sur place : le déplacement d'un tailleur se comprendrait mal pour un élément qui ne réclame aucune adaptation au terrain.

Seconde remarque : aussi curieux qu'il paraisse, ce bloc constitue le seul témoin de la technologie massaliole dans le domaine oléicole. Ni à Marseille ni à sa proximité immédiate, n'ont été retrouvés d'éléments d'huilerie qu'on puisse dater de la période préromaine : pour l'ensemble de la Gaule méridionale, on dénombre seulement trois sites, Entremont, la Courtine d'Ollioules près de 'Toulon ${ }^{8}$ et Mouriès, dans les Alpilles ${ }^{9}$. Les machineries attestées par ces trouvailles sont de type évolué, semblables aux installations italiennes de même époque ${ }^{10}$.

7 Ibid., p. 1.13.

\& J. Layet, La Courtine d'Ollioules, capilale aygricole de la fulure région lonlonnaise entre le VII $\mathrm{e}$ el la fin du $I I^{*}$ siècle auanl nolre ère, Toulon, 1949; Id., Le sancluaire cello-ligure, l'huilerie el le nouveau quartier de maisons de loppidum de la Courtine, Toulon, 1949. Ont ite trouves sur ce site cing maies dalles de: pressage; et trois contrepoids de treuil.

9 Inédil. Renseignement P. Arcelin. Il s'agit d'une maie en remploi, en pierre de Fontvieille.

10 J.-P. Brun, op. cil., p. 305. 

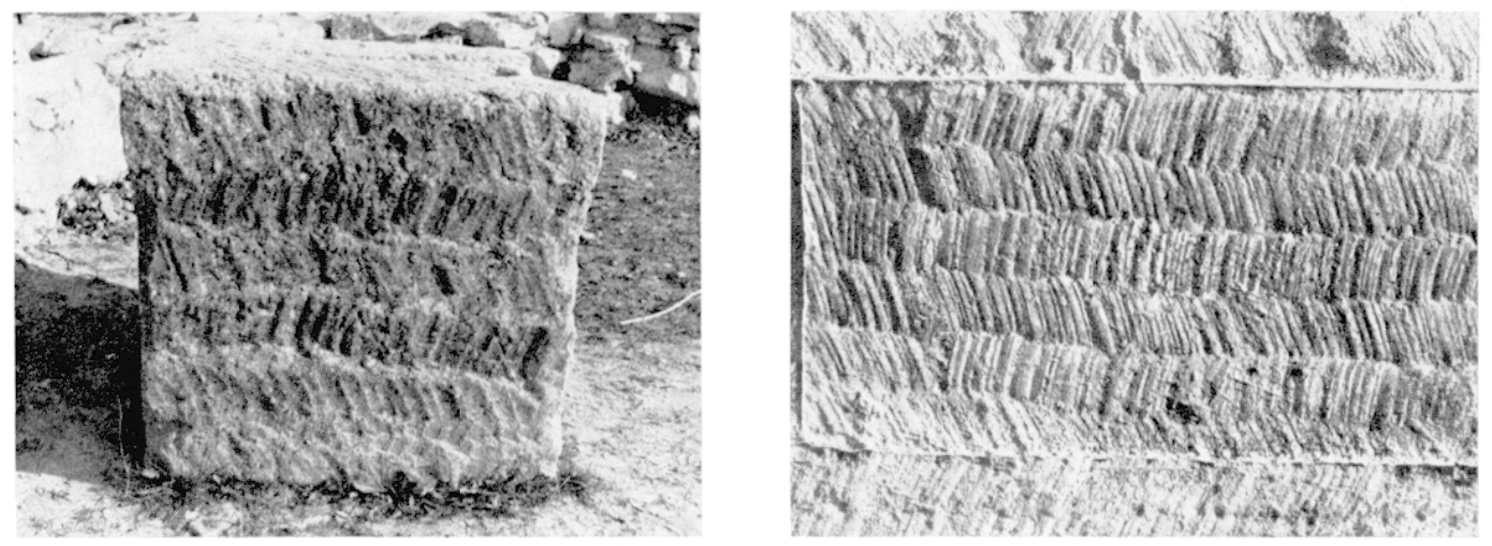

3 et 1 Détail de la taille de la pierre. A g., le bloc d'Entremont. A dr., un bloc du rempart de Marseille 3 : cliché de l'auleur. t : cliche J. Prodhomme.

Apres ces constalations, on entre dans un domaine plus risqué où il convient de contròler l'imagination. Line huilerie de fabrication massaliote dans la capitale des salyens: comment interpréter? On songe d'inslinct à la phrase de Justin sur l'enseignement donné par les Massaliotes aux indigènes : "tailler la vigne et planter l'olivier" (XI.III, 4), enseignement qui, comme il est logique, devait aller jusqu'à la technique de l'extraction de l'huile. Le point curieux, malgré tout, tient à l'origine géologique du contrepoids : pourquoi un transport d'environ $50 \mathrm{~km} \mathrm{--} \mathrm{du} \mathrm{Cap} \mathrm{Couronne} \mathrm{à} \mathrm{Entremont} \mathrm{...}$ alors que des carrieres aixoises, comme le notail F. Benoît, pouvaient convenir? Marseille vendait-elle des huileries en pièces détachées?

Yous avons suggéré en d'autres lieux que la cilé phocéenne avail pu faire commerce de sa lechnologie, aussi bien "civile» que "mililaire " ${ }^{11}$. En l'occurrence, le pressoir d'Entremont illustre de manière concrete le " transfert " en dehors du domaine territorial de Marseille (ou des zones qu'elle pouvait contrôler) non seulement de son savoir mais des appareils qu'elle fabriquait. Qu'il s'agisse d'un exemple unique n'a guère d'importance : ce type de pièces ne se retrouve qu'assez rarement. D'autre part, le soin - si on ose dire ... qui fut apporté, lors de la première destruction

11 "Marseilles, Rome and Gaul from the third to the first century B,C. ". Trade in the ancient ecommm!, Londres, 1983, p. 76-86.
d'Entremont' ${ }^{12}$, au démantèlement des huileries et, notamment, au bris des contrepoids des treuils, laisse supposer l'importance économique attachée à ces installations ${ }^{13}$.

\section{Christian Golmaxal}

\section{Post-scriplum.}

Cette note elait rédigé lorsque nous avons appris la mort de Robert Ambard all cours de l'été 1983. Robert Ambard, collaborateur technique au cixRs, fut le second de fr. Benoît sur bien des chantiers de la region provençale : Muscapeu (Var, villa des Grassi Aix al, au premier chef, Entremont dont, en fait, il controilait quotidiennement le déroulement des fouilles. C'ótnit le meilleur connaisseur des antiquités d'Aix. Il représentait la mémoire de mille découvertes 'ju'il narrait aree gentillesse. L'arrêt des foulles d'Entremont lui causa un profond désenchantement. Bien des pages, 'fu'il ne signa jamais, lui doivent leur substance. Fin sourenir de nombreuses visites communes à Entremont, nous dédions ce qui précède à la mémoire d'un archéologue aussi passionné que discret, aussi érudil que génereux.

12 Seules, quatre maies ont éte retrouvées en place, trois par les fouilles de F. Benoit, une par des fouilles plus récentes inédites!. Tous les contrepoids, on l'a dit, sont lirises at deplaces. I.e remploi de nombreuses pieces dans des murs on comme supports de toiture atteste que la destruction qui les affecta ne fut pas la dernière subie par le site. Faut-il supposer que les deux campagnes de 124 et de 123 touchèrent la capitale des Salyens et que ces remplois démontrent une reconstruction opérée entre les deux sièges? Lne reprise extensive et sérieuse des fouilles répondra, espérons-le, dans un avenir proche.

13 Ce qui exclut, semble-t-il, que ces huileries aient été possédées par les tenants du parti promassaliote que nous signale Diodore (XXXIY, 23'. 